\title{
Quantum witness and invasiveness of cosmic neutrino measurements
}

\author{
P. Kurashvili@ ${ }^{1}$ L. Chotorlishvili $\odot,{ }^{2}$ K. A. Kouzakov $\odot,{ }^{3}$ A. G. Tevzadze $\odot,{ }^{4}$ and A. I. Studenikin ${ }^{5,6}$ \\ ${ }^{1}$ National Centre for Nuclear Research, Warsaw 00-681, Poland \\ ${ }^{2}$ Institute für Physik, Martin-Luther Universität Halle-Wittenberg, D-06120 Halle/Saale, Germany \\ ${ }^{3}$ Department of Nuclear Physics and Quantum Theory of Collisions, Faculty of Physics, \\ Lomonosov Moscow State University, Moscow 119991, Russia \\ ${ }^{4}$ Kutaisi International University, Kutaisi University Campus, Kutaisi 4600, Georgia \\ ${ }^{5}$ Department of Theoretical Physics, Faculty of Physics, Lomonosov Moscow State University, \\ Moscow 119991, Russia \\ ${ }^{6}$ Joint Institute for Nuclear Research, Dubna 141980, Moscow Region, Russia
}

(Received 3 August 2020; accepted 1 February 2021; published 16 February 2021)

\begin{abstract}
Measurements of cosmic neutrinos have a reach potential for providing an insight into fundamental neutrino properties. For this a precise knowledge about an astrophysical environment of cosmic neutrinos propagation is needed. However this is not always possible, and the lack of information can bring about theoretical uncertainties in our physical interpretation of the results of experiments on cosmic neutrino fluxes. We formulate an approach that allows one to quantify the uncertainties using the apparatus of quantum measurement theory. We consider high-energy Dirac neutrinos emitted by some distant source and propagating towards Earth in the interstellar space. We take into account the neutrino magnetic moment interaction with stochastic interstellar magnetic fields and describe the neutrino evolution in the formalism of the Lindblad master equation for the neutrino density matrix. It is supposed that neutrinos on their way to Earth pass through highly magnetized dense layers of a cosmic object that "stops" left-handed neutrinos and lets only sterile, right-handed neutrinos to propagate farther. Such a filter mimics the strongest effect on the neutrino flux that can be induced by the cosmic object and that can be missed in the theoretical interpretation of the lab measurements due to the insufficient information about the astrophysical environment of the neutrino propagation. Treating the neutrino interaction with the neutrino filter of the cosmic object as the first, neutrino-spin measurement, whose result is not recorded, we study its invasive effect on the second, neutrino-flavor measurement in the lab. We quantify the invasiveness of the first, blind measurement by means of quantum witness that in the discussed case has an advantage over the well-known Leggett-Garg inequality, since the latter explores two-time correlation functions of the same operator. We solve analytically the Lindblad master equation for time evolution of the neutrino density matrix and on this basis we calculate the quantum witness for measuring cosmic electron neutrinos in the lab. We present numerical illustrations of the robust invasive effect showing that the quantum witness as a function of the distance between Earth and a cosmic object can be an asymptotically nonvanishing quantity despite the thermalization of the neutrino spin induced by stochastic interstellar magnetic fields.
\end{abstract}

DOI: 10.1103/PhysRevD.103.036011

\section{COSMIC NEUTRINOS AND THE CONCEPT OF QUANTUM MEASUREMENT}

Many objects in the Universe produce vast amount of cosmic neutrinos with different energies. These include particles from a low-energy $(\sim 10 \mathrm{MeV})$, a high-energy $(>100 \mathrm{MeV})$, to an extremely high-energy $(>1 \mathrm{PeV})$ or even an ultrahigh-energy $(>1 \mathrm{EeV})$ range. Neutrinos carry

Published by the American Physical Society under the terms of the Creative Commons Attribution 4.0 International license. Further distribution of this work must maintain attribution to the author(s) and the published article's title, journal citation, and DOI. Funded by SCOAP ${ }^{3}$. unique information about the internal environment of highenergy astrophysical objects that are not normally directly observable.

It is known that $99 \%$ of the energy of a core collapse supernova explosion is carried away by a neutrino flux. All these particles should contribute to the diffuse supernova neutrino background [1] that is not yet detected. Still the first albeit only detection of a neutrino burst from a single supernova explosion was detected for the 1987 event that occurred $51 \mathrm{kpc}$ away [2]. The neutrino (antineutrino) flux from this extragalactic explosion arrived before the visible light of the explosion reached Earth. Neutrinos from the core of the protoneutron star have traveled away from a strongly magnetized interior and were emitted toward Earth. 
Detection of 25 neutrinos in this experiment gave birth to neutrino astronomy. Three decades later, neutrino detectors have improved significantly. It is estimated that we would detect tens of thousands of neutrinos if a supernova similar to the 1987 event [3] were to explode today. Today, it is possible to estimate neutrino signals as progenitors of supernova explosions for neutrino observatories, including JUNO [4], DUNE [5], Hyper-Kamiokande [6], and IceCube [7]. We have already been waiting a decade for a supernova explosion in our cosmic neighborhood-an event that is expected to occur a few times a century. Neutrino flux from such an event will give us a bonanza of astrophysical and particle physics information [8] that will significantly advance our understanding of high-energy astrophysical phenomena.

Magnetic moments of the neutrinos emitted from the corecollapse supernova should be aligned toward the magnetic field axis of the developing neutron star. Strongest polarization should occur for neutrinos emitted from magnetars, where a magnetic field can reach up to $10^{14}-10^{15} \mathrm{G}$. These objects are developed from at least $10 \%$ of the core collapse supernova and are known for having the strongest magnetic fields in the Universe [9].

Even stronger flux of magnetically polarized neutrinos should originate from the neutron star mergers. Two cases of this phenomenon, long thought to be an exotic event, have already been detected using the gravitational wave signal [10]. Simultaneous detection of the gravitational wave and neutrino flux will allow us to employ these particles in multimessenger astronomy. The list of exotic events producing high-energy neutrino fluxes in strong magnetic fields may include hypothesized quark nova and collapsars.

At extra high-energy scales, neutrinos are born during gamma ray bursts from hypernova [11]. Similar energies are expected to be emitted by quasars in the active galactic nuclei, where supermassive black holes create relativistic outflows $[12,13]$. With much higher uncertainties of local properties of these objects, it is clear that a strong magnetic field should be present near the active area [14]. Thus, these luminous sources of neutrinos can be detected for extragalactic distances exceeding $1 \mathrm{Gpc}$.

Neutrino fluxes reaching us from such distant sources are influenced by galactic and extragalactic magnetic fields. These weak fields can exert a dissipative effect on the neutrino spin polarization by their stochastic components. Although microscopic in amplitudes these fields affect particles at cosmic distances and can have a significant cumulative effect. Magnetic fields in our Galaxy do not exceed $4 \mu \mathrm{G}$ on average [15], although they are stronger at the Galactic Center.

Magnetic fields are detected at extragalactic scales as well. These fields can reach $10^{-9} \mathrm{G}$ amplitudes at $1 \mathrm{Mpc}$ scale [16]. Assuming a turbulent spectrum of extragalactic magnetic fields, we may estimate a small-scale stochastic component to reach up to $0.3 \mu \mathrm{G}$ amplitudes [17]. These weak fields can affect ultrahigh-energy neutrinos that travel up to Gpc distances.

From the plethora of cosmic neutrino emitting objects, we focus on such sources that can be found both in the lowenergy and in the high-energy as well as in extra highenergy intervals. Below, we consider evolution of neutrinos that are emitted from a distant source and traverse the cosmic space diluted with the stochastic magnetic fields until they reach the detector in the lab. While propagating in a magnetic field, neutrinos experience spin and spin-flavor oscillations due to a nonzero neutrino magnetic moment $[18,19]$. For simplicity we limit ourselves with the case of two neutrino flavors $\left(\nu_{e}\right.$ and $\left.\nu_{\mu}\right)$.

In this paper we propose a new tool for the theoretical analysis of cosmic neutrino measurements, which is based on the theoretical framework for quantum witness experiments. For this we define two consequent quantum measurements of a neutrino. Figure 1 shows a sketch illustration of the quantum witness experiment using cosmic neutrinos. Suppose that neutrinos propagate away from their source, in central areas of high-energy cosmic objects. Neutrinos pass through the outer shell of a highly magnetized dense environment, where left-handed neutrinos are deflected or absorbed, and mainly right-handed neutrinos pass to travel towards Earth. Setting the first deflection event as a quantum spin measurement, we may use neutrino flavor detection in the lab as the second measurement. The absence of information about the results of the first measurement implies that the measurement was blind. The blind measurement has no invasive effect on the neutrino state.

Often high energy cosmic objects are covered by a neutrinosphere-a layer transparent for neutrinos. In this case, both left- as well as right-handed neutrinos will propagate away from the source. In the opposite limit, active areas of cosmic objects covered by highly magnetized dense material that can deflect left-handed neutrinos and create spin asymmetry in the emitted neutrino flux can serve as a "cosmic neutrino filter" around astrophysical sources.

We describe the measurements by the positive-operator valued measure projectors. The first projector measurement is the essence of the quantum Maxwell Demon [20], which holds the information, whether neutrinos of particular types get in and out of the cosmic filter. The second projector measurement is a typical neutrino experiment in the lab that defines the neutrino flavor. However, the result of the second measurement incisively depends on the outcome of the first measurement: our interpretation of the result (for example, such as the origin of neutrinos, the initial neutrino flux, its flavor and spectral composition, etc.) can be critically affected by whether Demon shares his results with us or not. In the latter case, the first measurement appears to be a blind measurement, i.e., its results are not recorded. Clearly, this mimics the situation when we do not have enough information about the conditions of cosmic neutrino propagation, in particular, about the presence of 


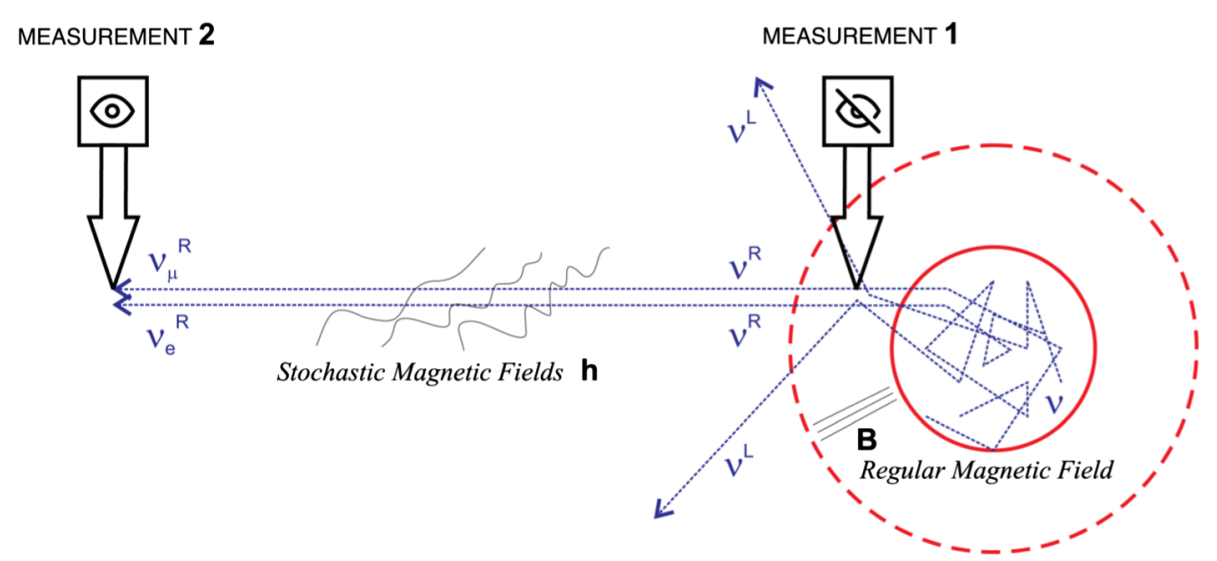

FIG. 1. Illustration of the quantum witness experiment using cosmic neutrino observations. Neutrinos propagating away from their source (i.e., a core-collapse supernova or a protoneutron object developed from a neutron star merger) pass through the outer highdensity environment of the cosmic object with extreme magnetic fields. Here left-handed neutrinos can be deflected, and only righthanded (anti)neutrinos propagate to be observed by neutrino detectors on Earth. Such a cosmic filter can be considered the first "invasive measurement," while the neutrino detection event as the second measurement. The first measurement has a quantum invasive effect on the second measurement, depending on the neutrino flux properties emitted from the source area. If information is not extracted through the first measurement, it has no invasive effect and is termed blind.

the cosmic neutrino filter. Since the lack of information can lead to an incorrect physical interpretation of cosmic neutrino measurements in the lab, the approach developed in this work allows us to quantify the credibility of our interpretation.

In what follows, we analyze cosmic neutrinos in the interstellar space filled with a magnetic field and explore the invasive effect of the cosmic neutrino filter on the neutrino measurement in the lab. The standard tool for analyzing the invasiveness of quantum measurements is the Leggett-Garg method. For neutrino physics, Leggett-Garg inequality was studied only recently in the case of unitary evolution [21]. However, in the case of open quantum systems, exploring the two-time correlation functions is a formidable problem [22], analytically not accessible for the neutrino problem. Therefore, as an alternative to the Legget-Garg method, we exploit the concept of quantum witness and the Novikov's dissipative channel [23].

It should be noted that the phenomenon of neutrino oscillations is closely related to the concept of quantum coherence. This concept touches upon the macroscopic coherence, i.e., superposition of a macroscopic large number of states and involves two core principles: (i) macroscopic realism per se argues that the preexisting value of the quantity in question can be inferred through the measurements done on the macroscopic system, (ii) noninvasive measurability means that one can perform measurements without distorting the state of the system [24-33]. In reality the situation is more complex. Except for specific initial states, the measurement has a backaction and induces noncommuting dynamical changes in an observable [34]. When the system initially is prepared in the superposition of two (or more) states $|\phi\rangle=a\left|\psi_{n}\right\rangle+b\left|\psi_{m}\right\rangle$, using the measurement operator $\hat{\prod}_{n}=\left|\psi_{n}\right\rangle\left\langle\psi_{n}\right|, n \in \mathcal{N}$, one cannot determine the state of the system without a destructive effect on the state (the invasive measurement). The quantum witness quantifies the invasiveness of the measurement. Up-to-date in quantum metrology mainly nonrelativistic quantum systems have been discussed. Nevertheless, the interest in studies of such relativistic systems as neutrinos has recently emerged $[21,27]$.

Below we elaborate on the general formalism for describing the quantum witness of the cosmic neutrino measurement and discuss its application in feasible neutrino experiments. We treat the neutrino evolution using the method based on the Lindblad master equation [35]. This approach is presently widely used in studies of neutrino quantum decoherence in different environments and under various experimental conditions (see, for instance, Refs. [36-48]).

The work is organized as follows. In Sec. II, the general formulation is presented. In Sec. III we define the quantum witness for the neutrino flavor measurement in the lab. Section IV is devoted to the analytical derivation of the quantum witness from the neutrino density matrix. In Sec. V, we give illustrations of the invasive effect of the cosmic neutrino filter on the neutrino flavor measurement in the lab. In Sec. VI we compare statistical dispersion for supernova neutrino detection with the quantum invasive effect. Section VII summarizes this work. In the appendixes, we deliver details of solving the Lindblad master equation for neutrino evolution.

\section{NEUTRINO EVOLUTION IN AN INTERSTELLAR MAGNETIC FIELD}

In the scope of our interest are two Dirac neutrino helicity basis states $\left|\nu_{1}^{ \pm}\right\rangle,\left|\nu_{2}^{ \pm}\right\rangle$with masses $m_{1}$ and $m_{2}$. For 
the sake of convenience we switch from the mass basis $\left(\nu_{1}^{+}, \nu_{1}^{-}, \nu_{2}^{+}, \nu_{2}^{-}\right)^{T}$ to the flavor basis $\left(\nu_{e}^{R}, \nu_{e}^{L}, \nu_{\mu}^{R}, \nu_{\mu}^{L}\right)^{T}$ :

$$
\begin{aligned}
& \left|\nu_{e}^{R, L}\right\rangle=\cos \theta\left|\nu_{1}^{ \pm}\right\rangle+\sin \theta\left|\nu_{2}^{ \pm}\right\rangle, \\
& \left|\nu_{\mu}^{R, L}\right\rangle=-\sin \theta\left|\nu_{1}^{ \pm}\right\rangle+\cos \theta\left|\nu_{2}^{ \pm}\right\rangle .
\end{aligned}
$$

The Hamiltonian of the problem is given by (see Refs. [49,50])

$$
\hat{H}_{\mathrm{eff}}=\hat{H}_{\mathrm{vac}}+\hat{H}_{B},
$$

where $\hat{H}_{\text {vac }}$ is the vacuum part

$\hat{H}_{\mathrm{vac}}=\omega_{\nu}\left(\begin{array}{cccc}-\cos 2 \theta & 0 & \sin 2 \theta & 0 \\ 0 & -\cos 2 \theta & 0 & \sin 2 \theta \\ \sin 2 \theta & 0 & \cos 2 \theta & 0 \\ 0 & \sin 2 \theta & 0 & \cos 2 \theta\end{array}\right)$,

with

$$
\omega_{\nu}=\frac{\Delta m^{2}}{4 E_{\nu}}, \quad \Delta m^{2}=m_{2}^{2}-m_{1}^{2}
$$

and $E_{\nu}$ being the neutrino energy. The Hamiltonian of the neutrino interaction with a magnetic field in the flavor representation can be presented as $[51,52]$

$$
H_{B}=\left(\begin{array}{cccc}
\left(\frac{\mu}{\gamma}\right)_{e e} B_{\|} & \mu_{e e} B_{\perp} & -\left(\frac{\mu}{\gamma}\right)_{e \mu} B_{\|} & \mu_{e \mu} B_{\perp} \\
\mu_{e e} B_{\perp} & -\left(\frac{\mu}{\gamma}\right)_{e e} B_{\|} & \mu_{e \mu} B_{\perp} & -\left(\frac{\mu}{\gamma}\right)_{e \mu} B_{\|} \\
-\left(\frac{\mu}{\gamma}\right)_{e \mu} B_{\|} & \mu_{e \mu} B_{\perp} & -\left(\frac{\mu}{\gamma}\right)_{\mu \mu} B_{\|} & \mu_{\mu \mu} B_{\perp} \\
\mu_{e \mu} B_{\perp} & -\left(\frac{\mu}{\gamma}\right)_{e \mu} B_{\|} & \mu_{\mu \mu} B_{\perp} & -\left(\frac{\mu}{\gamma}\right)_{\mu \mu} B_{\|}
\end{array}\right),
$$

where $B_{\|}$and $B_{\perp}$ are the parallel and transverse magneticfield components with respect to the neutrino velocity, and the magnetic moments $\mu_{\ell \ell^{\prime}}$ and $(\mu / \gamma)_{\ell \ell^{\prime}}\left(\ell, \ell^{\prime}=e, \mu\right)$ are related to those in the mass representation $\mu_{j k}(j, k=1,2)$ as follows:

$$
\begin{aligned}
& \mu_{e e}=\mu_{11} \cos ^{2} \theta+\mu_{22} \sin ^{2} \theta+\mu_{12} \sin 2 \theta \\
& \mu_{e \mu}=\mu_{12} \cos 2 \theta+\frac{1}{2}\left(\mu_{22}-\mu_{11}\right) \sin 2 \theta \\
& \mu_{\mu \mu}=\mu_{11} \sin ^{2} \theta+\mu_{22} \cos ^{2} \theta-\mu_{12} \sin 2 \theta
\end{aligned}
$$

and

$$
\begin{aligned}
& \left(\frac{\mu}{\gamma}\right)_{e e}=\frac{\mu_{11}}{\gamma_{1}} \cos ^{2} \theta+\frac{\mu_{22}}{\gamma_{2}} \sin ^{2} \theta+\frac{\mu_{12}}{\gamma_{12}} \sin 2 \theta, \\
& \left(\frac{\mu}{\gamma}\right)_{e \mu}=\frac{\mu_{12}}{\gamma_{12}} \cos 2 \theta+\frac{1}{2}\left(\frac{\mu_{22}}{\gamma_{2}}-\frac{\mu_{11}}{\gamma_{1}}\right) \sin 2 \theta, \\
& \left(\frac{\mu}{\gamma}\right)_{\mu \mu}=\frac{\mu_{11}}{\gamma_{1}} \sin ^{2} \theta+\frac{\mu_{22}}{\gamma_{2}} \cos ^{2} \theta-\frac{\mu_{12}}{\gamma_{12}} \sin 2 \theta .
\end{aligned}
$$

Here $\gamma_{1}$ and $\gamma_{2}$ are the Lorenz factors of the massive neutrinos, and

$$
\frac{1}{\gamma_{12}}=\frac{1}{2}\left(\frac{1}{\gamma_{1}}+\frac{1}{\gamma_{2}}\right)
$$

We consider the case when the galactic and extragalactic magnetic fields are composed of the large-scale regular component $\vec{B}$ that enters Eq. (5) and a small-scale stochastic component $\vec{h}$.

The stochastic magnetic field $\vec{h}$ is a result of interstellar fluctuations, galactic winds, cosmic turbulence, and primordial magnetic field fluctuations. It is characterized by the correlation function [53] $\left\langle h_{\alpha}(t) h_{\beta}(0)\right\rangle=\frac{w^{2}}{2 \mu_{\nu}^{2}} \delta(t)$, where $\mu_{\nu}$ is a putative neutrino magnetic moment and $w^{2}=k_{B} T$, with $T$ being the effective temperature.

The density matrix of the system obeys the Lindblad master equation [35] in the form

$$
\frac{d \hat{\varrho}}{d t}=-i[\hat{H}, \hat{\varrho}]-\frac{w^{2}}{2}\left(\hat{\varrho} \hat{V}^{2}+\hat{V}^{2} \hat{\varrho}-2 \hat{V} \hat{\varrho} \hat{V}\right) .
$$

In the most general case the $\hat{V}$ matrix in Eq. (9) is given by

$$
V_{i k}=\left\langle i\left|\hat{I}^{\left(\nu_{e}\right)} \otimes \hat{v}^{\left(\nu_{\mu}\right)}+\hat{I}^{\left(\nu_{\mu}\right)} \otimes \hat{v}^{\left(\nu_{e}\right)}\right| k\right\rangle,
$$

where $|i\rangle$ and $|k\rangle(i, k=1,2,3,4)$ are the eigenstates of the Hamiltonian $\hat{H}_{\text {eff }}$ (see Ref. [49] for details). $\hat{I}^{\left(\nu_{\ell}\right)}$ is the $2 \times 2$ unit matrix acting in the Hilbert space of the $\nu_{\ell}$ neutrino. The $2 \times 2$ matrix $\hat{v}^{\left(\nu_{\ell}\right)}$ also acts in the Hilbert space of the $\nu_{\ell}$ neutrino. It can be presented as

$$
\hat{v}^{\left(\nu_{\ell}\right)}=v_{0} \hat{I}+\vec{v} \cdot \hat{\vec{\sigma}}
$$

where $\hat{\vec{\sigma}}$ is the Pauli vector. Below we utilize the following parametrization:

$$
\vec{v}=(v \sin \beta \cos \alpha, v \sin \beta \sin \alpha, v \cos \beta) .
$$

In its general form the dissipator (10) describes relaxation of both transverse and longitudinal neutrino spin components. However, in the particular case $\beta=\pi / 2$, the cosmic magnetic field does not thermalize the $\sigma_{z}$ component of the neutrino spin. 
We analytically solve Eq. (9) in the eigenbasis of the Hamiltonian (2). The solution $\hat{\varrho}(t)$ is cumbersome and is presented in Appendix A.

\section{QUANTUM WITNESS OF COSMIC NEUTRINO MEASUREMENTS}

As was already mentioned, if neutrinos pass through the cosmic neutrino filter they acquire a preferential helicity polarization, since the high-density matter filters out the left-handed neutrinos. This process can be described by the positive-operator valued measure (POVM) projectors, projecting the neutrino state on the direction of the neutrino flux propagation.

Let us first consider the case when neutrinos pass through a neutrino filter (we call this "the first propagation scheme"). The initial neutrino state at $t=0$, i.e., just before entering the filter, is $|\phi\rangle$. In the general case the efficient quantum measurement of neutrino spin polarization transforms this state into the postmeasurement state

$$
|\Phi\rangle=\frac{\left(\hat{\Pi}_{\vec{s}} \otimes \hat{I}^{(\ell)}\right)|\phi\rangle}{\sqrt{\left\langle\phi\left|\left(\hat{\Pi}_{\vec{s}} \otimes \hat{I}^{(\ell)}\right)\right| \phi\right\rangle}},
$$

where $\vec{s}$ is a unit vector of spin polarization of the neutrino on the way out of the neutrino filter and $\hat{I}^{(\ell)}$ is the identity operator acting on the flavor space. Taking into account that the left-handed neutrinos are mostly filtered-out by the cosmic object, the postmeasurement density matrix is given by

$$
\hat{\varrho}_{\text {post }}=|\Phi\rangle\langle\Phi|=\frac{\left(\hat{\Pi}_{+} \otimes \hat{I}^{(\ell)}\right) \hat{\varrho}\left(\hat{I}^{(\ell)} \otimes \hat{\Pi}_{+}\right)}{\operatorname{Tr}\left(\left(\hat{\Pi}_{+} \otimes \hat{I}^{(\ell)}\right) \hat{\varrho}\left(\hat{I}^{(\ell)} \otimes \hat{\Pi}_{+}\right)\right)},
$$

where $\hat{\varrho}=|\phi\rangle\langle\phi|$ is the initial density matrix and the positive-helicity projector operator is

$$
\hat{\Pi}_{+}=\frac{1}{2}\left(1+\frac{\vec{p} \vec{\sigma}}{|\vec{p}|}\right)
$$

After measuring helicity we evolve the density matrix through the trace-preserving Novikov's map $\hat{\mathcal{F}}\left[\hat{\varrho}_{\text {post }}\right]$ that mimics the effect of a stochastic magnetic field in Eq. (9). The second measurement is then performed by detecting the active flavor neutrino state $\left|\nu_{\ell}^{L}\right\rangle$ in the lab. We describe this detection procedure through the projector operator $\hat{\Pi}_{\nu_{\ell}^{L}}=\hat{\Pi}_{-} \hat{\Pi}_{\ell}$, where the negative-helicity projector operator reads

$$
\hat{\Pi}_{-}=\frac{1}{2}\left(1-\frac{\vec{p} \vec{\sigma}}{|\vec{p}|}\right)
$$

and the flavor projector operator is $\hat{\Pi}_{\ell}=|\ell\rangle\langle\ell|, \ell=e, \mu$. Thus, the probability of detecting the active flavor neutrino state $\left|\nu_{\ell}^{L}\right\rangle$ is given by

$$
\mathcal{Q}_{L}^{(\ell)}=\operatorname{Tr}\left\{\hat{\Pi}_{\nu_{\ell}^{L}} \hat{\mathcal{F}}\left[\hat{\varrho}_{\text {post }}\right]\right\} .
$$

We now consider the second propagation scheme, meaning that the neutrinos do not meet the neutrino filter and pass through a transparent neutrinosphere on their way from the source to the detector. In this case, the neutrino flavor state is measured without preliminary measurement of the neutrino helicity. The probability of detecting the active flavor neutrino state $\left|\nu_{\ell}^{L}\right\rangle$ is given by

$$
\mathcal{P}_{L}^{(\ell)}=\operatorname{Tr}\left\{\hat{\Pi}_{\nu_{\ell}^{L}} \hat{\mathcal{F}}[\hat{\varrho}]\right\}
$$

The difference between the two neutrino propagation schemes is due to the invasive effect of the neutrino helicity measurement "performed" by the cosmic neutrino filter. For quantifying the invasiveness of the neutrino measurement in the lab we use the quantum witness

$$
\mathcal{W}_{L}^{(\ell)}=\left|\mathcal{P}_{L}^{(\ell)}-\mathcal{Q}_{L}^{(\ell)}\right|
$$

Note that quantum witness ranges from 0 to 1 , so that the value of 0 corresponds to no invasive effect and that of 1 to a maximal invasive effect of the first measurement performed by the neutrino filter. Accordingly, the confidence in the interpretation of the result of the second measurement is either maximal or minimal depending on whether quantum witness (19) equals 0 or 1 .

\section{QUANTUM WITNESS AND THE DENSITY MATRIX}

In the mass basis, the entire $4 \times 4$ density matrix can be presented in the conventional form

$$
\hat{\varrho}=\left(\begin{array}{ll}
\varrho^{(1)} & \varrho^{(2)} \\
\varrho^{(3)} & \varrho^{(4)}
\end{array}\right),
$$

where $Q^{(\alpha=1,2,3,4)}$ are the matrices of dimension $2 \times 2$. The elements of the four quadrants can be enumerated with separate sets of indices 1 and 2, corresponding to the spinup and spin-down states, respectively. We expand the quadrants over the basis of Pauli matrices:

$$
\varrho^{(\alpha)}=r_{0}^{(\alpha)} I+\vec{\sigma} \vec{r}^{(\alpha)} .
$$

The elements of matrices $\varrho^{(\alpha)}$ are linked to the respective coefficients $r_{i=0,1,2,3}^{(\alpha)}$ through the following relations:

$$
\begin{aligned}
& \varrho_{11}^{(\alpha)}=r_{0}^{(\alpha)}+r_{3}^{(\alpha)}, \\
& \varrho_{22}^{(\alpha)}=r_{0}^{(\alpha)}-r_{3}^{(\alpha)}, \\
& \varrho_{12}^{(\alpha)}=r_{1}^{(\alpha)}-i r_{2}^{(\alpha)},
\end{aligned}
$$




$$
\varrho_{21}^{(\alpha)}=r_{1}^{(\alpha)}+i r_{2}^{(\alpha)}
$$

In these variables, the entire Lindblad equation for the density matrix splits into a set of four independents linear systems. Each quadrant contains four elements and admits the exact analytical solution $r_{i}^{(\alpha)}(t)$. We evolve the density matrix through the trace-preserving dissipative channel, conserving the entire density matrix's trace. Therefore the sum of traces of the diagonal quadrants $\varrho^{(1)}$ and $\varrho^{(4)}$ is the integral of motion.

The measurement is the essence of the action of flavor and spin projection matrices on the density matrix. In terms of Eq. (20), the effect of the application of the spin projection operator is expressed as the action of the operator $\hat{\Pi}_{ \pm}$on both sides of the $2 \times 2$ matrix minors $\varrho^{(\alpha)}$, where $\alpha$ is either 1 or 4 :

$$
\begin{aligned}
\hat{\Pi}_{\vec{s}} Q^{(\alpha)}(0) \hat{\Pi}_{\vec{s}}= & \frac{1}{4}(1+\vec{\sigma} \vec{s})\left[r_{0}^{(\alpha)}(0) I+\vec{\sigma} \vec{r}^{(\alpha)}(0)\right] \\
& \times(1+\vec{\sigma} \vec{s}) \\
= & \frac{1+\vec{\sigma} \vec{s}}{2}\left[r_{0}^{(\alpha)}(0)+\vec{r}^{(\alpha)}(0) \vec{s}\right] .
\end{aligned}
$$

The respective traces read

$$
\begin{aligned}
N_{+} & =\operatorname{Tr}\left(\hat{\Pi}_{+} \varrho^{(1)}(0) \hat{\Pi}_{+}\right)+\operatorname{Tr}\left(\hat{\Pi}_{+} \varrho^{(4)}(0) \hat{\Pi}_{+}\right) \\
& =r_{0}^{(1)}(0)+r_{3}^{(1)}(0)+r_{0}^{(4)}(0)+r_{3}^{(4)}(0) \\
& =\frac{1}{2}+\left(r_{3}^{(1)}(0)+r_{3}^{(4)}(0)\right)
\end{aligned}
$$

and

$$
\begin{aligned}
N_{-} & =\operatorname{Tr}\left(\hat{\Pi}_{-} \varrho(0) \hat{\Pi}_{-}\right)+\operatorname{Tr}\left(\hat{\Pi}_{-} \varrho^{(4)}(0) \hat{\Pi}_{-}\right) \\
& =r_{0}^{(1)}(0)-r_{3}^{(1)}(0)+r_{0}^{(4)}(0)-r_{3}^{(4)}(0) \\
& =\frac{1}{2}-\left(r_{3}^{(1)}(0)+r_{3}^{(4)}(0)\right) .
\end{aligned}
$$

Note that in terms of components of the full $4 \times 4$ matrix, $\hat{\varrho}$, $N_{+}=\hat{\varrho}_{11}(0)+\hat{\varrho}_{33}(0), N_{-}=\hat{\varrho}_{22}(0)+\hat{\varrho}_{44}(0)$.

The postmeasurement density matrix quadrants are equal to

$$
\begin{aligned}
\varrho_{\text {post }}^{(\alpha)}(0) & =\frac{1}{2} \frac{\left(r_{0}^{(\alpha)}(0)+r_{3}^{(\alpha)}(0)\right)\left(1+\sigma_{z}\right)}{N_{+}} \\
& =\frac{r_{0}^{(\alpha)}(0)+r_{3}^{(\alpha)}(0)}{1+2\left(r_{3}^{(1)}(0)+r_{3}^{(4)}(0)\right)}\left(1+\sigma_{z}\right) .
\end{aligned}
$$

The postmeasurement density matrices $\varrho_{\text {post }}^{(\alpha)}$ obey the equation of motion but for different initial conditions as compared to the case when the neutrino density matrix is not filtered out through the first helicity measurement. The coefficient $r_{0}^{(\alpha)}$ of the expansion in Eq. (21) is, in essence, the trace of a $2 \times 2$ matrix $Q^{(\alpha)}$ and is conserved in time.

Let us derive the projection operators for the electron neutrino in explicit form:

$$
\begin{aligned}
\hat{\Pi}_{\nu_{e}^{R, L}}= & \left|\nu_{e}^{R, L}\right\rangle\left\langle\nu_{e}^{R, L}\right| \\
= & \left(\cos \theta\left|\nu_{1}^{ \pm}\right\rangle+\sin \theta\left|\nu_{2}^{ \pm}\right\rangle\right) \\
& \times\left(\left\langle\nu_{1}^{ \pm}\right| \cos \theta+\left\langle\nu_{2}^{ \pm}\right| \sin \theta\right) .
\end{aligned}
$$

Following the same recipe, one can derive the POVM projectors for the muon neutrino. Therefore we deduce for the flavor projectors in the mass basis:

$$
\hat{\Pi}_{e}=\left(\begin{array}{cc}
c_{\nu}^{2} & c_{\nu} s_{\nu} \\
c_{\nu} s_{\nu} & s_{\nu}^{2}
\end{array}\right)
$$

and

$$
\hat{\Pi}_{\mu}=\left(\begin{array}{cc}
s_{\nu}^{2} & -c_{\nu} s_{\nu} \\
-c_{\nu} s_{\nu} & c_{\nu}^{2}
\end{array}\right)
$$

where we introduced the notations $c_{\nu}=\cos \theta, s_{\nu}=\sin \theta$. In what follows, we exploit the flavor projection operators in the form

$$
\begin{aligned}
& \hat{\Pi}_{e}=\frac{1}{2}\left(1+\sigma_{1} \sin 2 \theta+\sigma_{3} \cos 2 \theta\right), \\
& \hat{\Pi}_{\mu}=\frac{1}{2}\left(1-\sigma_{1} \sin 2 \theta-\sigma_{3} \cos 2 \theta\right) .
\end{aligned}
$$

We insert these operators into the expression for the result in the second measurement scheme (18):

$$
\begin{aligned}
\mathcal{P}_{L}^{(\ell)} & =\operatorname{Tr}\left\{\hat{\Pi}_{\nu_{\ell}^{L}} \hat{\mathcal{F}}[\hat{\varrho}]\right\}=\operatorname{Tr}\left\{\hat{\Pi}_{\nu_{\ell}^{L}} \hat{\varrho}(t) \hat{\Pi}_{\nu_{\ell}^{L}}\right\} \\
& =\operatorname{Tr}\left\{\hat{\Pi}_{-} \hat{\Pi}_{\ell} \hat{\varrho}(t) \hat{\Pi}_{\ell} \hat{\Pi}_{-}\right\}
\end{aligned}
$$

Employing the density matrix in the form Eq. (20) and applying the spin and flavor projection operators given by Eqs. (16), (31), and (32), respectively, we obtain

$$
\begin{aligned}
\mathcal{P}_{L}^{(e)}(t)= & c_{\nu}^{2}\left[r_{0}^{(1)}(t)-r_{3}^{(1)}(t)\right]+s_{\nu}^{2}\left[r_{0}^{(4)}(t)-r_{3}^{(4)}(t)\right] \\
& +c_{\nu} s_{\nu}\left[r_{0}^{(2)}(t)+r_{0}^{(3)}(t)-r_{3}^{(2)}(t)-r_{3}^{(3)}(t)\right] \\
= & r_{0}^{(e)}(t)-r_{3}^{(e)}(t)
\end{aligned}
$$

where

$$
r_{i}^{(e)}(t)=c_{\nu}^{2} r_{i}^{(1)}(t)+s_{\nu}^{2} r_{i}^{(4)}(t)+c_{\nu} s_{\nu}\left[r_{i}^{(2)}(t)+r_{i}^{(3)}(t)\right],
$$


for $i=0,1,2,3$. For the case of measuring active muon neutrinos we get

$$
\mathcal{P}_{L}^{(\mu)}(t)=r_{0}^{(\mu)}(t)-r_{3}^{(\mu)}(t),
$$

where

$$
r_{i}^{(\mu)}(t)=s_{\nu}^{2} r_{i}^{(1)}(t)+c_{\nu}^{2} r_{i}^{(4)}(t)-c_{\nu} s_{\nu}\left[r_{i}^{(2)}(t)+r_{i}^{(3)}(t)\right] .
$$

The result in the case of the first measurement scheme (17) has the form

$$
\mathcal{Q}_{L}^{(\ell)}=\operatorname{Tr}\left\{\hat{\Pi}_{\nu_{\ell}^{L}} \hat{\mathcal{F}}\left[\hat{\varrho}_{\text {post }}\right]\right\}=\operatorname{Tr}\left\{\hat{\Pi}_{-} \hat{\Pi}_{\ell} \hat{\varrho}_{\text {post }}(t) \hat{\Pi}_{\ell} \hat{\Pi}_{-}\right\} .
$$

The final expressions are formally identical to Eqs. (36) and (38). However, the functions related to the quadrants of the density matrix, $\rho_{i}^{(\alpha)}(t)$, must be replaced by the functions corresponding to the postmeasurement matrix $\hat{\varrho}_{\text {post }}(t)$.

Since at $t=0$ only the right-handed electron neutrino component is different from zero, the functions $r_{\text {post }, 0}^{(1)}(t)$, $r_{\text {post }, 3}^{(1)}(t)$ obey the same equation of motion, but with different initial conditions:

$$
r_{\text {post }, 0}^{(1)}(0)=r_{\text {post }, 3}^{(1)}(0)=\frac{r_{0}^{(1)}(0)+r_{3}^{(1)}(0)}{1+2\left(r_{3}^{(1)}(0)+r_{3}^{(4)}(0)\right)} .
$$

The same holds for $r_{\text {post }, 0}^{(4)}(0)$ and $r_{\text {post }, 3}^{(4)}(0)$. Note that the sum in the parentheses in the denominator can be presented as

$$
r_{3}^{(1)}(0)+r_{3}^{(4)}(0)=r_{3}^{(e)}(0)+r_{3}^{(\mu)}(0)
$$

Hence

$$
\begin{aligned}
r_{\text {post }, 0}^{(e)}(0)= & c_{\nu}^{2} r_{\text {post }, 0}^{(1)}(0)+s_{\nu}^{2} r_{\text {post }, 0}^{(4)}(0) \\
& +s_{\nu} c_{\nu}\left[r_{\text {post }, 0}^{(2)}(0)+r_{\text {post }, 0}^{(3)}(0)\right],
\end{aligned}
$$

where

$$
r_{\text {post }, 0}^{(2)}(0)+r_{\text {post }, 0}^{(3)}(0)=\frac{r_{0}^{(2)}(0)+r_{0}^{(3)}(0)}{1+2\left(r_{3}^{(e)}(0)+r_{3}^{(\mu)}(0)\right)} .
$$

Using the derived results for $\mathcal{P}_{L}^{(e)}$ and $\mathcal{Q}_{L}^{(e)}$ in the expression for the quantum witness (19), we get

$$
\mathcal{W}_{L}^{(e)}=\left|r_{0}^{(e)}(t)-r_{3}^{(e)}(t)-r_{\mathrm{post}, 0}^{(e)}(t)+r_{\mathrm{post}, 3}^{(e)}(t)\right| .
$$

The time evolution of the density matrix $\hat{\varrho}$ and factors $r_{i}^{(\alpha)}$ follow the solution of the equation of neutrino motion and Novikov's map in Eq. (9). The details of solving analytically the Lindblad equation and, in particular, deriving the minors of the density matrix $\hat{\varrho}$ in the eigenbasis of the Hamiltonian (2) are presented in Appendixes B and $\mathrm{C}$.

\section{ILLUSTRATION OF THE INVASIVE EFFECT}

In the present work, we study the effect of two sequential measurements done on neutrinos traversing the interstellar/ extragalactic space. Due to the cosmic magnetic fields, propagation of a massive neutrino is accompanied by spinflavor oscillations, while the stochastic component of these fields has a random influence on neutrino spin polarization and leads to the decoherence effect.

We aim at examining the invasive effect of the first, blind measurement, namely the effective neutrino spin filtering by the cosmic object, on the result of the second measurement of an active neutrino performed in the lab on Earth. For this purpose we consider two measurement schemes: we filter out left-handed neutrinos in the cosmic neutrino filter and then measure its active flavor state at the lab, or measure the active flavor state directly, without spin filtering. Note that the measurement that performs the cosmic neutrino filter is blind because its result is not recorded. For classical systems, the first blind measurement is always noninvasive. The difference between results recorded in the lab in the cases of the first and second neutrino propagation schemes is entirely a quantum phenomenon and we quantify it through the quantum witness (19).

Below we illustrate the invasive effect of the first, blind measurement, assuming $\mu_{11}=\mu_{22}=\mu_{12}=\mu_{\nu}$ and that the energy of the neutrino magnetic moment interaction with an interstellar magnetic field is $\mu_{\nu} B=10^{-32} \mathrm{eV}$. This energy corresponds to the putative magnetic moment, $\mu_{\nu} \approx 4 \times 10^{-20} \mu_{B}$, that agrees with the value predicted for the Dirac neutrino by the minimally extended standard model [54],

$$
\mu_{\nu} \approx 3.2 \times 10^{-19}\left(\frac{m_{\nu}}{1 \mathrm{eV}}\right) \mu_{B},
$$

taking into account current upper bounds on the neutrino mass $m_{\nu}$ (see, for instance, Refs. [55,56]). When describing the influence of the stochastic magnetic field $\vec{h}$ in the Lindblad master equation (9), without loss of generality, we exploit the parametrization (12) of the dissipator term with a unit vector length $v=1$ and a zero angle $\alpha=0$. Further, from Eq. (12) it follows that if $\alpha=0$ the matrix $\hat{V}$ given by Eq. (10) is real, and only $v_{1,3}$ components enter in the dissipator term, which now depends on the parameter $w^{2}$ and the angle $\beta$. The Lindblad equation parameter $w^{2}$ characterizes the strength of the dissipation and is usually equal to some fraction of the energy of the interaction with 


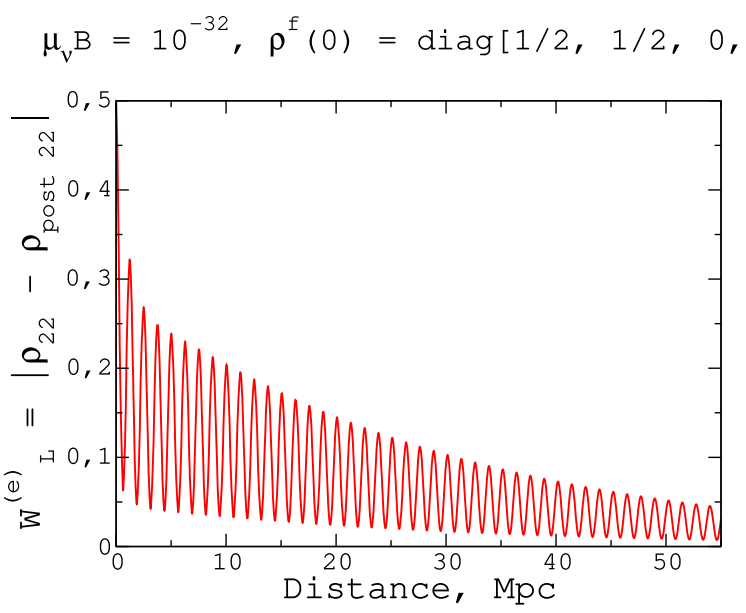

FIG. 2. The quantum witness for detecting left-handed electron neutrinos at the lab depending on the distance from the cosmic object to Earth. In the initial state, there are only electron neutrinos with equal fractions of left- and right-handed particles: $r_{0}^{(e)}(0)=1 / 2, r_{3}^{(e)}(0)=0$.

the magnetic field $\vec{B}$. Here we use a reasonable value $w^{2}=0.1 \mu_{\nu} B$. For the angle $\beta$ we use a value of $\pi / 4$, implying that both neutrino longitudinal and transverse spin components are thermalized due to the stochastic magnetic field on equal footing.

Figure 2 shows the quantum witness (45) as a function of the distance between the cosmic object and Earth when the neutrino flux at $t=0$ (i.e., at the moment of time corresponding to the neutrino passing through the cosmic filter in the first propagation scheme) consists only of electron neutrinos. It is assumed in Fig. 2 that the neutrinos have already traveled a large distance before being filtered, so their spins have been fully thermalized. For this reason, we set $r_{3}^{(e)}(0)=0$, meaning that the numbers of right- and left-handed neutrinos entering the cosmic spin filter are equal. The shown quantum witness function decays, exhibiting oscillations, and tends to zero at large distances. The latter means that the invasive effect of the cosmic filter on the result of the measurement performed in the lab vanishes. Such a decaying behavior of the quantum witness is not general. In Fig. 3 we show the quantum witness (45) when the density matrix describing the neutrino flux before entering the cosmic spin filter is fully thermalized in the flavor basis. It can be seen that the quantum witness function has now a more complex behavior, which is different from "quantum beats" observed in Fig. 2. First, it decays only at small distances. Second, it also exhibits oscillations, but the oscillation pattern shows a superposition of two functions that oscillate with the same or almost the same frequency but in antiphase: the one function is decaying and the other is growing. The observed picture is explained by the behaviors of the probabilities $\mathcal{Q}_{L}^{(e)}$ and $\mathcal{P}_{L}^{(e)}$ of measuring the active electron neutrino at $\mu_{v} B=10^{-32}, \rho^{f}(0)=\operatorname{diag}[1 / 4,1 / 4,1 / 4,1 / 4]$

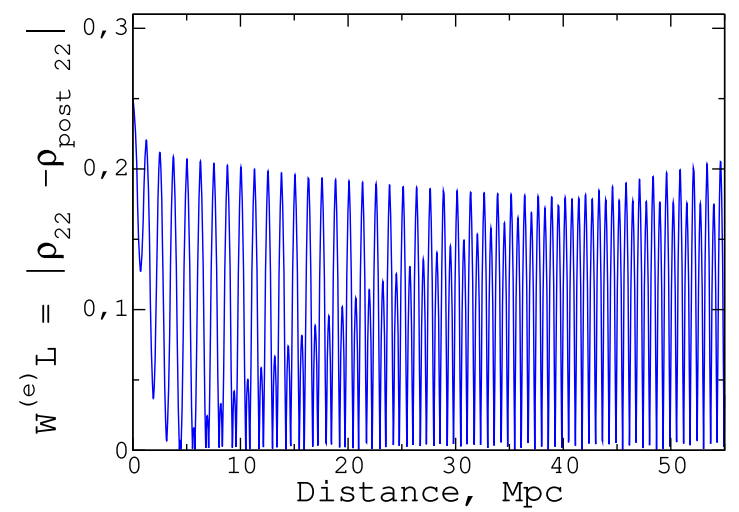

FIG. 3. The same as in Fig. 2, but when in the initial state the density matrix in the flavor basis is given by $\hat{\varrho}=\operatorname{diag}(1 / 4$, $1 / 4,1 / 4,1 / 4)$.

the lab in the first and second propagation schemes, respectively.

\section{THE STATISTICAL DISPERSION VS QUANTUM UNCERTAINTY}

Information extracted through the detection of the neutrino ensemble has a statistical character. Statistical dispersion of the ensemble data can be broader than the invasive effect of the quantum measurement. Analysis of the quantum uncertainty can be important when its effect is larger compared to the statistical dispersion of the neutrino flux measurements. In this section, we compare the statistical distribution of the ensemble data with the effect of quantum witness and show that for the standard parameters that mimic experimentally feasible neutrino detectors, the effect of quantum witness can be substantial.

For illustrative purposes, we discuss the case of supernova explosions producing tremendous outbursts of neutrinos of energies of several $\mathrm{MeV}$. If such an event happens in our galaxy, the neutrinos can be detected in considerable numbers by the current neutrino detectors. As it was shown through theoretical models, $99 \%$ of the gravitational binding energy of the core-collapse supernova is emitted in the form of neutrinos of all flavors. Total energy of such a neutrino flux can reach up to $10^{53}$ erg energy levels. Still, most neutrinos emitted from such sources fall in the 12-16 MeV energy range. If a supernova occurs in our cosmic neighborhood at distances up to $10 \mathrm{kpc}$, the neutrino flux produced by it will be substantial [57]. Moreover, neutrino detectors today already have sufficiently highenergy resolution to provide high precision reconstruction of the neutrino spectra from nearby supernova sources.

For the majority of present date detectors the most common reaction for the neutrino-type particle detection is the inverse beta decay with interacting electron antineutrino and a proton inside the detector, $\overline{\nu_{e}}+p \rightarrow e^{+}+n$. 
Other, more rare events include elastic neutrino-proton and neutrino-electron scattering, or even neutral-current interactions with ${ }^{12} \mathrm{C}$ nuclei. Although it is still characterized by a tiny cross section, in IceCube, Super-Kamiokande, and future large neutrino telescopes JUNO [58] and HyperKamiokande [59], it will be possible to detect jointly up to 90,000 such events if the supernova event similar to SN1987a occurs at $50 \mathrm{kpc}$ distance today [60]. Moreover, it is expected that we will be able to reconstruct neutrino spectra for supernova explosions in the nearby galaxies, up to the Mpc scales [61].

Monte Carlo supernova simulations with synthetic data indicate the capacity of modern neutrino detectors to analyze neutrino flux spectrum with high precision: it is estimated that the energy spectrum of the incoming neutrino flux can be reconstructed with statistical errors well below $10 \%$ in the $10-30 \mathrm{MeV}$ energy range. Direct comparison with a quantum witness experiment (see Fig. 2) shows that the invasive effect of the cosmic neutrino measurement set by outer layers of the distant supernova can exceed statistical error of the neutrino energy spectrum measurements at distances not exceeding $10 \mathrm{Mpc}$. This estimate reveals that the effect of quantum uncertainty induced by a cosmic neutrino filter is detectable for supernova explosions inside our galaxy or even in a local group of galaxies that span distances up to $3 \mathrm{Mpc}$.

\section{SUMMARY}

In this work we have developed a new approach for interpretation and analysis of the data of experiments with cosmic neutrinos. Our approach employs the concepts of invasiveness and quantum witness that are used in the theory of quantum measurements. We have considered two subsequent measurements of neutrinos from a distant source traversing the interstellar and intergalactic space and interacting with stochastic cosmic magnetic fields along the propagation trajectory. The first measurement is performed by a neutrino filter of the dense cosmic object, which effectively absorbs active, left-handed neutrinos, thus letting only right-handed neutrinos to propagate further to Earth. The second measurement is performed in the lab at Earth and is a typical measurement of active flavor neutrinos. The first measurement is blind and has an invasive effect on the result of the second measurement, undermining the credibility of our physical interpretation of the results obtained in the lab. In order to quantify this credibility, we defined the quantum witness for the neutrino measurement in the lab and obtained analytical expressions for the quantum witness in terms of the elements of the neutrino density matrix. Using an analytical solution of the Lindblad master equation for the neutrino density matrix, we demonstrated the invasive effect of the cosmic filter on the neutrino measurement in the lab. We showed that the quantum witness can exhibit qualitatively different behaviors for different properties of the neutrino flux that meets the neutrino filter. In particular, the decay of the quantum witness as a function of the distance from the cosmic object that one may naively expect due to the neutrino spin thermalization effects is not observed when the density matrix of the neutrino flux meeting the neutrino filter has in the flavor basis a diagonal form with all four neutrino states equally populated. Our approach can be used for quantifying theoretical uncertainties associated with the lack of information about the conditions of cosmic neutrinos propagation when interpreting and analyzing the results of experiments with neutrinos from distant astrophysical sources.

\section{ACKNOWLEDGMENTS}

The work of K. A. K. and A.I.S. is supported by the Russian Foundation for Basic Research under Grant No. 20-52-53022-GFEN-A.

\section{APPENDIX A: THE LINDBLAD MASTER EQUATION}

In what follows, we use the eigenbasis $\left(\tilde{\nu}_{1}, \tilde{\nu}_{2}, \tilde{\nu}_{3}, \tilde{\nu}_{4}\right)^{T}$ of the Hamiltonian (2) (see Ref. [49]). In this representation the Lindblad master equation (9) takes the form

$$
\begin{aligned}
\frac{d \rho_{n m}}{d t}= & -i\left(E_{n}-E_{m}\right) \varrho_{m n}-\frac{w^{2}}{2} \sum_{q}\left(\rho_{n q} V_{q m}^{2}+V_{n q}^{2} \varrho_{q m}\right) \\
& +w^{2} \sum_{q, s} V_{n q} \varrho_{q s} V_{s m},
\end{aligned}
$$

where $E_{1,2,3,4}$ are the eigenvalues of the Hamiltonian (2).

We present the density matrix as

$$
\hat{\varrho}=\left(\begin{array}{ll}
\varrho^{(1)} & \varrho^{(2)} \\
\varrho^{(3)} & \varrho^{(4)}
\end{array}\right),
$$

where $\varrho^{(\alpha)}$ are the $2 \times 2$ minors.

Then in Eq. (A1) one has the expressions of the form

$$
\sum_{q=1}^{2}\left(v_{n q}^{\left(\nu_{\ell}\right) 2} \varrho_{q m}^{(\alpha)}+R_{n q}^{(\alpha)} v_{q m}^{\left(\nu_{\ell}\right) 2}\right),
$$

and

$$
\sum_{q, s=1}^{2} v_{n q}^{\left(\nu_{\ell}\right)} R_{q s}^{(\alpha)} v_{s m}^{\left(\nu_{\ell}\right)}
$$

for the first and second sums, respectively. All $\varrho^{(\alpha)}$ along with $v^{\left(\nu_{\ell}\right)}$ are expanded in the basis of the three Pauli matrices and $2 \times 2$ unit matrix:

$$
R^{(\alpha)}=r_{0}^{(\alpha)} I+\vec{r}^{(\alpha)} \cdot \vec{\sigma}
$$


and

$$
v^{\left(\nu_{\ell}\right)}=v_{0} I+\vec{v} \cdot \vec{\sigma}
$$

with

$$
\begin{aligned}
r_{0}^{(\alpha)} & =\frac{1}{2}\left(\varrho_{11}^{(\alpha)}+\varrho_{22}^{(\alpha)}\right), \\
r_{1}^{(\alpha)} & =\frac{1}{2}\left(\varrho_{12}^{(\alpha)}+\varrho_{21}^{(\alpha)}\right), \\
r_{2}^{(\alpha)} & =\frac{i}{2}\left(\varrho_{12}^{(\alpha)}-\varrho_{21}^{(\alpha)}\right), \\
r_{3}^{(\alpha)} & =\frac{1}{2}\left(\varrho_{11}^{(\alpha)}-\varrho_{22}^{(\alpha)}\right) .
\end{aligned}
$$

Analogous relations are used between the elements of the matrix $v$ and coefficients of its expansion.

We now expand Eqs. (A3) and (A4) and exploit Eqs. (A6) and (A5):

$$
\begin{aligned}
& \sum_{q=1}^{2}\left(v_{n q}^{\left(\nu_{\ell}\right) 2} \varrho_{q m}^{(\alpha)}+\varrho_{n q}^{(\alpha)} v_{q m}^{\left(\nu_{\ell}\right) 2}\right) \\
& =2\left[\left(v_{0}^{2}+v^{2}\right) r_{0}^{(\alpha)}+2 v_{0} \vec{v} \cdot \vec{r}^{(\alpha)}\right] I_{n m} \\
& +4 v_{0} r_{0}^{(\alpha)} \vec{v} \cdot \vec{\sigma}_{n m}+2\left(v_{0}^{2}+v^{2}\right) \vec{r}^{(\alpha)} \cdot \vec{\sigma}_{n m}, \\
& \sum_{q, s=1}^{2} v_{n q}^{\left(\nu_{\ell}\right)} \varrho_{q s}^{(\alpha)} v_{s m}^{\left(\nu_{\ell}\right)} \\
& =\left[\left(v_{0}^{2}+v^{2}\right) r_{0}^{(\alpha)}+2 v_{0} \vec{v} \cdot \vec{r}^{(\alpha)}\right] I_{n m} \\
& \quad+2\left[v_{0} r_{0}^{(\alpha)}+\vec{v} \cdot \vec{r}^{(\alpha)}\right] \vec{v} \cdot \vec{\sigma}_{n m} \\
& \quad+\left(v_{0}^{2}-v^{2}\right) \vec{r}^{(\alpha)} \cdot \vec{\sigma}_{n m} .
\end{aligned}
$$

Summing up Eqs. (A11) and (A12) with the same weights as in Eq. (A1), one gets the following dissipative contribution:

$$
\begin{aligned}
L^{(\alpha)}= & -\frac{w^{2}}{2} \sum_{q=1}^{2}\left(v_{n q}^{\left(\nu_{\ell}\right) 2} \varrho_{q m}^{(\alpha)}+\varrho_{n q}^{(\alpha)} v_{q m}^{\left(\nu_{\ell}\right) 2}\right) \\
& +w^{2} \sum_{q, s=1}^{2} v_{n q}^{\left(\nu_{\ell}\right)} \varrho_{q s}^{(\alpha)} v_{s m}^{\left(\nu_{\ell}\right)} \\
= & 2 w^{2}\left[\left(\vec{v} \cdot \vec{r}^{(\alpha)}\right) \vec{v} \cdot \vec{\sigma}_{n m}-v^{2} \vec{r}^{(\alpha)} \cdot \vec{\sigma}_{n m}\right] .
\end{aligned}
$$

Equation (A13) is also decomposed in the basis of $2 \times 2$ matrices:

$$
L^{(\alpha)}=\Lambda_{0}^{(\alpha)} I+\vec{\Lambda}^{(\alpha)} \cdot \vec{\sigma},
$$

where

$$
\begin{gathered}
\Lambda_{0}^{(\alpha)}=0, \\
\Lambda_{i}^{(\alpha)}=2 w^{2}\left[\left(\vec{v} \cdot \vec{r}^{(\alpha)}\right) v_{i}-v^{2} r_{i}^{(\alpha)}\right] .
\end{gathered}
$$

Let us derive the equations for the elements of the minor $\varrho^{(1)}$. Using Eqs. (A5), (A7), (A11), (A12), (A13), and (A1), one gets

$$
\begin{gathered}
\frac{d}{d t} \varrho_{11}=\frac{d}{d t}\left(r_{0}^{(1)}+r_{3}^{(1)}\right)=\Lambda_{3}^{(1)}, \\
\frac{d}{d t} \varrho_{22}=\frac{d}{d t}\left(r_{0}^{(1)}-r_{3}^{(1)}\right)=-\Lambda_{3}^{(1)}, \\
\frac{d}{d t} \varrho_{12}=\frac{d}{d t} r_{-}^{(1)}=-i \omega_{12} r_{-}^{(1)}+\Lambda_{-}^{(1)}, \\
\frac{d}{d t} \varrho_{21}=\frac{d}{d t} r_{+}^{(1)}=-i \omega_{21} r_{+}^{(1)}+\Lambda_{+}^{(1)},
\end{gathered}
$$

where $r_{ \pm}=r_{1} \pm i r_{2}, \quad \Lambda_{ \pm}^{(1)}=\Lambda_{1}^{(1)} \pm i \Lambda_{2}^{(1)}, \quad$ and $\omega_{12}=$ $E_{1}-E_{2}=-\omega_{21}$. Note that the sum of the diagonal matrix elements $\varrho_{11}+\varrho_{22}=r_{0}^{(1)}$ is constant in time. The set of equations for $\varrho^{(4)}$ is obtained from Eqs. (A17)-(A20) by changing $r^{(1)}$ to $r^{(4)}$ and $\omega_{12}$ to $\omega_{34}$. The sum $\varrho_{33}+\varrho_{44}=$ $r_{0}^{(4)}$ is also conserved as well as the complete trace of the density matrix.

The set of equations for the minor $\varrho^{(2)}$ reads

$$
\begin{aligned}
\frac{d}{d t} Q_{13} & =\frac{d}{d t}\left(r_{0}^{(2)}+r_{3}^{(2)}\right) \\
& =-i \omega_{13}\left(r_{0}^{(2)}+r_{3}^{(2)}\right)+\Lambda_{3}^{(2)}, \\
\frac{d}{d t} \varrho_{24} & =\frac{d}{d t}\left(r_{0}^{(2)}-r_{3}^{(2)}\right) \\
& =-i \omega_{24}\left(r_{0}^{(2)}-r_{3}^{(2)}\right)-\Lambda_{3}^{(2)}, \\
\frac{d}{d t} \varrho_{14} & =\frac{d}{d t} r_{-}^{(2)}=-i \omega_{14} r_{-}^{(2)}+\Lambda_{-}^{(2)} \\
\frac{d}{d t} \varrho_{23} & =\frac{d}{d t} r_{+}^{(2)}=-i \omega_{23} r_{+}^{(2)}+\Lambda_{+}^{(2)} .
\end{aligned}
$$

The equations for $\varrho^{(3)}$ are obtained from Eqs. (A21)-(A24) upon Hermitian conjugation.

\section{APPENDIX B: THE SOLUTION FOR $\mathrm{a}^{(1)}$}

Below we consider the system of equations for the minor $\varrho^{(1)}$, omitting upper indexes in $r^{(\alpha)}$ and designating $\omega=\omega_{12}=-\omega_{21}$. After redefinition of the time variable

$$
\tau=2 w^{2} t
$$


the equations acquire the following form:

$$
\begin{gathered}
\frac{d}{d \tau} r_{0}=0, \\
\frac{d}{d \tau} r_{+}=\left[\frac{v_{+} v_{-}}{2}-v_{3}^{2}-i \bar{\omega}\right] r_{+}+\frac{v_{+}^{2}}{2} r_{-} \\
+v_{+} v_{3} r_{3}, \\
\frac{d}{d \tau} r_{-}=\frac{v_{-}^{2}}{2} r_{+}+\left[-\frac{v_{+} v_{-}}{2}-v_{3}^{2}+i \bar{\omega}\right] r_{-} \\
+v_{-} v_{3} r_{3}, \\
\frac{d}{d \tau} r_{3}=v_{-} v_{3} \frac{r_{+}}{2}+v_{+} v_{3} \frac{r_{-}}{2}-v_{+} v_{-} r_{3},
\end{gathered}
$$

where $\bar{\omega}=\omega / 2 w^{2}$.

In the case of $v^{2}=1$ and $v_{y}=0$, one has $\vec{v}=$ $(\sin \beta, 0, \cos \beta)$ and it is convenient to rewrite the system of equations in terms of $r_{1,2}$ instead of $r_{ \pm}$:

$$
\begin{gathered}
\frac{d}{d \tau} r_{0}=0, \\
\frac{d}{d \tau} r_{1}=-r_{1} \cos ^{2} \beta+r_{2} \bar{\omega}+r_{3} \sin \beta \cos \beta, \\
\frac{d}{d \tau} r_{2}=-r_{1} \bar{\omega}-r_{2} \cos ^{2} \beta, \\
\frac{d}{d \tau} r_{3}=r_{1} \sin \beta \cos \beta-r_{3} \sin ^{2} \beta .
\end{gathered}
$$

To solve the above system, one must diagonalize the $3 \times 3$ matrix:

$$
\mathcal{M}^{(1)}=\left(\begin{array}{ccc}
-\cos ^{2} \beta & \bar{\omega} & \sin \beta \cos \beta \\
-\bar{\omega} & -\cos ^{2} \beta & 0 \\
\sin \beta \cos \beta & 0 & -\sin ^{2} \beta
\end{array}\right) .
$$

The general solution of the system is a sum of exponents:

$$
r_{i}(\tau)=\sum_{k=1}^{3} C_{i k} e^{i \nu_{k} \tau}
$$

where $\nu_{i}$ are the eigenvalues of the above matrix and the integration constants are given by the following expressions:

$$
\begin{aligned}
C_{i 1} & =\frac{B_{0 i} \nu_{2} \nu_{3}-B_{1 i}\left(\nu_{2}+\nu_{3}\right)+B_{2 i}}{\left(\nu_{1}-\nu_{2}\right)\left(\nu_{1}-\nu_{3}\right)}, \\
C_{i 2} & =\frac{B_{0 i} \nu_{1} \nu_{3}-B_{1 i}\left(\nu_{1}+\nu_{3}\right)+B_{2 i}}{\left(\nu_{2}-\nu_{1}\right)\left(\nu_{2}-\nu_{3}\right)},
\end{aligned}
$$

$$
C_{i 3}=\frac{B_{0 i} \nu_{1} \nu_{2}-B_{1 i}\left(\nu_{1}+\nu_{2}\right)+B_{2 i}}{\left(\nu_{3}-\nu_{1}\right)\left(\nu_{3}-\nu_{2}\right)},
$$

where

$$
\begin{gathered}
B_{0 i}=r_{i}(0), \\
B_{1 i}=\sum_{k=1}^{3} \mathcal{M}_{i k}^{(1)} r_{k}(0), \\
B_{2 i}=\sum_{k, l=1}^{3} \mathcal{M}_{i k}^{(1)} \mathcal{M}_{k l}^{(1)} r_{l}(0) .
\end{gathered}
$$

The equations for $\varrho^{(4)}$ are similar to Eqs. (B2)-(B5), except that one must replace $\omega=\omega_{12}$ with $\omega=\omega_{34}=E_{3}-E_{4}$. Note that there is a condition of the trace conservation:

$$
\operatorname{Tr} \varrho=\sum_{i} \varrho_{i i}=r_{0}^{(1)}+r_{0}^{(4)}=1 .
$$

Another limitation is that the density matrix should be Hermitian, $\varrho_{12}=\varrho_{21}^{\dagger}, \varrho_{34}=\varrho_{43}^{\dagger}$, which is already satisfied by (B12)-(B15).

\section{APPENDIX C: THE SOLUTION FOR $\varrho^{(2)}$}

The system of equations for the minor $\varrho^{(2)}$ is given by

$$
\begin{gathered}
\frac{d}{d \tau} r_{0}=-\frac{i}{2}\left(\bar{\omega}_{13}+\bar{\omega}_{24}\right) r_{0}-\frac{i}{2}\left(\bar{\omega}_{13}-\bar{\omega}_{24}\right) r_{3}, \\
\frac{d}{d \tau} r_{+}=\left[-\frac{v_{+} v_{-}}{2}-v_{3}^{2}-i \bar{\omega}_{23}\right] r_{+} \frac{v_{+}^{2}}{2} r_{-} \\
+v_{+} v_{3} r_{3},
\end{gathered}
$$

$$
\begin{aligned}
\frac{d}{d \tau} r_{-}= & \frac{v_{-}^{2}}{2} r_{+}+\left[-\frac{v_{+} v_{-}}{2}-v_{3}^{2}-i \bar{\omega}_{14}\right] r_{-} \\
& +v_{-} v_{3} r_{3},
\end{aligned}
$$

$$
\begin{aligned}
\frac{d}{d \tau} r_{3}= & -\frac{i}{2}\left(\bar{\omega}_{13}-\bar{\omega}_{24}\right) r_{0}+\frac{v_{-} v_{3}}{2} r_{+} \\
& +\frac{v_{+} v_{3}}{2} r_{-}+\left[-\frac{i}{2}\left(\bar{\omega}_{13}+\bar{\omega}_{24}\right)-v_{+} v_{-}\right] r_{3} .
\end{aligned}
$$

Using the ansatz $\vec{v}=(\sin \beta, 0, \cos \beta)$ and changing to $r_{1}$ and $r_{2}$, one gets the system for $\varrho^{(2)}$ as

$$
\begin{gathered}
\frac{d}{d \tau} r_{0}=-i \bar{\omega}_{+} r_{0}-i \bar{\omega}_{-} r_{3}, \\
\frac{d}{d \tau} r_{1}=\left(-\cos ^{2} \beta-i \bar{\omega}_{+}\right) r_{1}+\bar{\omega}_{0} r_{2} \\
+r_{3} \sin \beta \cos \beta,
\end{gathered}
$$




$$
\begin{aligned}
\frac{d}{d \tau} r_{2}= & -\bar{\omega}_{0} r_{1}+\left(-\cos ^{2} \beta-i \bar{\omega}_{+}\right) r_{2}, & (\mathrm{C} 7) & \bar{\omega}_{0}=\frac{\bar{\omega}_{12}+\bar{\omega}_{34}}{2} \\
\frac{d}{d \tau} r_{3}= & -i \bar{\omega}_{-} r_{0}+r_{1} \sin \beta \cos \beta & & \bar{\omega}_{ \pm}=\frac{\bar{\omega}_{13} \pm \bar{\omega}_{24}}{2} . \\
& -\left(i \omega_{+}+\sin ^{2} \beta\right) r_{3} . & (\mathrm{C} 8) &
\end{aligned}
$$

The frequencies in the above formulas are

Solving the system requires diagonalization of the $4 \times 4$ matrix:

$$
\mathcal{M}_{4}^{(2)}=\left(\begin{array}{cccc}
-i \bar{\omega}_{+} & 0 & 0 & -i \bar{\omega}_{-} \\
0 & -\cos ^{2} \beta-i \bar{\omega}_{+} & \bar{\omega}_{0} & \sin \beta \cos \beta \\
0 & -\bar{\omega}_{0} & -\cos ^{2} \beta-i \bar{\omega}_{+} & 0 \\
-i \bar{\omega}_{-} & \sin \beta \cos \beta & 0 & -\sin ^{2} \beta-i \omega_{+}
\end{array}\right)
$$

The final solution is given in the form of a linear combination of exponential functions:

$$
r_{i}^{(2)}(\tau)=\sum_{k=1}^{4} C_{i k} e^{\nu_{k} \tau}
$$

with the integration constants

$$
\begin{gathered}
C_{i 1}=\frac{-B_{0 i} \nu_{2} \nu_{3} \nu_{4}+B_{1 i}\left(\nu_{2} \nu_{3}+\nu_{2} \nu_{4}+\nu_{3} \nu_{4}\right)-B_{2 i}\left(\nu_{2}+\nu_{3}+\nu_{4}\right)+B_{3 i}}{\left(\nu_{1}-\nu_{2}\right)\left(\nu_{1}-\nu_{3}\right)\left(\nu_{1}-\nu_{4}\right)}, \\
C_{i 2}=\frac{B_{0 i} \nu_{1} \nu_{3} \nu_{4}-B_{1 i}\left(\nu_{1} \nu_{3}+\nu_{1} \nu_{4}+\nu_{3} \nu_{4}\right)+B_{2 i}\left(\nu_{1}+\nu_{3}+\nu_{4}\right)+B_{3 i}}{\left(\nu_{2}-\nu_{1}\right)\left(\nu_{2}-\nu_{3}\right)\left(\nu_{2}-\nu_{4}\right)}, \\
C_{i 3}=\frac{-B_{0 i} \nu_{1} \nu_{2} \nu_{4}+B_{1 i}\left(\nu_{1} \nu_{2}+\nu_{1} \nu_{4}+\nu_{2} \nu_{4}\right)-B_{2 i}\left(\nu_{1}+\nu_{2}+\nu_{4}\right)+B_{3 i}}{\left(\nu_{3}-\nu_{1}\right)\left(\nu_{3}-\nu_{2}\right)\left(\nu_{3}-\nu_{4}\right)}, \\
C_{i 4}=\frac{B_{0 i} \nu_{1} \nu_{2} \nu_{3}-B_{1 i}\left(\nu_{1} \nu_{2}+\nu_{1} \nu_{3}+\nu_{2} \nu_{3}\right)+B_{2 i}\left(\nu_{1}+\nu_{2}+\nu_{3}\right)+B_{3 i}}{\left(\nu_{4}-\nu_{1}\right)\left(\nu_{4}-\nu_{2}\right)\left(\nu_{4}-\nu_{1}\right)},
\end{gathered}
$$

where

$$
\begin{gathered}
B_{0 i}=r_{i}(0), \\
B_{1 i}=\sum_{k=1}^{3} \mathcal{M}_{i k}^{(2)} r_{k}(0), \\
B_{2 i}=\sum_{k, l=1}^{3} \mathcal{M}_{i k}^{(2)} \mathcal{M}_{k l}^{(2)} r_{l}(0), \\
B_{3 i}=\sum_{k, l, m=1}^{3} \mathcal{M}_{i k}^{(2)} \mathcal{M}_{k l}^{(2)} \mathcal{M}_{l m}^{(2)} r_{m}(0) .
\end{gathered}
$$


[1] H. Zhang et al. (The Super-Kamiokande Collaboration), Astropart. Phys. 60, 41 (2015).

[2] K. Hirata et al. (Kamiokande-II Collaboration), Phys. Rev. Lett. 58, 1490 (1987); R. M. Bionta et al., Phys. Rev. Lett. 58, 1494 (1987).

[3] M. G. Aartsen et al. (IceCube-Gen2 Collaboration), arXiv: 2008.04323.

[4] F. An et al., J. Phys. G 43, 030401 (2016).

[5] C. Adams et al., arXiv:1307.7335v3.

[6] K. Abe et al., arXiv:1805.04163v2.

[7] S. Seadrow, A. Burrows, D. Vartanyan, D. Radice, and M. A. Skinner, Mon. Not. R. Astron. Soc. 480, 4710 (2018).

[8] G. G. Raffelt, Prog. Part. Nucl. Phys. 64, 393 (2010).

[9] V. M. Kaspi and A. M. Beloborodov, Annu. Rev. Astron. Astrophys. 55, 261 (2017).

[10] B. P. Abbott et al. (LIGO Scientific, Virgo Collaborations), Astrophys. J. Lett. 892, L3 (2020).

[11] X.-Y. Wang, S. Razzaque, P. Meszaros, and Z.-G. Dai, Phys. Rev. D 76, 083009 (2007).

[12] A. Aab et al. J. Cosmol. Astropart. Phys. 06 (2020) 017.

[13] O. Kalashev, D. Semikoz, and I. Tkachev, JETP Lett. 120, 541 (2015).

[14] W. Kollatschny, N. Schartel, M. Zetzl, M. Santos-Lleo, P. M. Rodriguez-Pascual, and L. Ballo, Astron. Astrophys. 577, L1 (2015).

[15] C. Sobey et al., Mon. Not. R. Astron. Soc. 484, 3646 (2019).

[16] P. A. R. Ade et al. (Planck Collaboration), Astron. Astrophys. 594, A19 (2016).

[17] A. Brandenburg, T. Kahniashvili, S. Mandal, A. R. Pol, A. G. Tevzadze, and T. Vachaspati, Phys. Rev. D 96, 123528 (2017).

[18] C. Giunti and A. Studenkin, Rev. Mod. Phys. 87, 531 (2015).

[19] C. Giunti, K. A. Kouzakov, Y.-F. Li, A. V. Lokhov, A. I. Studenikin, and S. Zhou, Ann. Phys. (Berlin) 528, 198 (2016).

[20] S. Deffner, Phys. Rev. E 88, 062128 (2013).

[21] J. A. Formaggio, D. I. Kaiser, M. M. Murskyj, and T. E. Weiss, Phys. Rev. Lett. 117, 050402 (2016).

[22] M. Ban, S. Kitajima, and F. Shibata, Phys. Rev. A 97, 052101 (2018).

[23] E. A. Novikov, JETP 20, 1290 (1965); A. Dutta, A. Rahmani, and A. del Campo, Phys. Rev. Lett. 117, 080402 (2016).

[24] C. Emary, N. Lambert, and F. Nori, Rep. Prog. Phys. 77, 039501 (2014).

[25] J. Hoffmann, C. Spee, O. Gühne, and C. Budroni, New J. Phys. 20, 102001 (2018).

[26] J. Kofler and C. Brukner, Phys. Rev. A 87, 052115 (2013).

[27] X.-K. Song, Y. Huang, J. Ling, and M.-H. Yung, Phys. Rev. A 98, 050302(R) (2018).

[28] J. J. Halliwell and C. Mawby, Phys. Rev. A 100, 042103 (2019).

[29] J. Naikoo, S. Banerjee, and A. M. Jayannavar, Phys. Rev. A 100, 062132 (2019).

[30] N. Nikitin and K. Toms, Phys. Rev. A 100, 062314 (2019).

[31] N. S. Williams and A. N. Jordan, Phys. Rev. Lett. 100, 026804 (2008).
[32] C. Robens, W. Alt, D. Meschede, C. Emary, and A. Alberti, Phys. Rev. X 5, 011003 (2015).

[33] B. Skinner, J. Ruhman, and A. Nahum, Phys. Rev. X 9, 031009 (2019).

[34] A. J. Leggett and A. Garg, Phys. Rev. Lett. 54, 857 (1985); G. Schild and C. Emary, Phys. Rev. A 92, 032101 (2015); K. Wang, G. C. Knee, X. Zhan, Z. Bian, J. Li, and P. Xue, Phys. Rev. A 95, 032122 (2017); D. Avis, P. Hayden, and M. M. Wilde, Phys. Rev. A 82, 030102(R) (2010); C. M. Li, N. Lambert, Y. N. Chen, G. Y. Chen, and F. Nori, Sci. Rep. 2, 885 (2012).

[35] G. Lindblad, Commun. Math. Phys. 48, 119 (1976).

[36] K. Stankevich and A. Studenikin, Phys. Rev. D 101, 056004 (2020).

[37] Y. Farzan, T. Schwetz, and A. Yu. Smirnov, J. High Energy Phys. 07 (2008) 067.

[38] E. Lisi, A. Marrone, and D. Montanino, Phys. Rev. Lett. 85, 1166 (2000).

[39] G. Barenboim and N. E. Mavromato, J. High Energy Phys. 01 (2005) 034.

[40] G. Barenboim, N. Mavromatos, S. Sarkar, and A. WaldronLauda, Nucl. Phys. B758, 90 (2006).

[41] F. Benatti and R. Floreanini, Phys. Rev. D 64, 085015 (2001).

[42] R. L. N. Oliveira, M. M. Guzzo, and P. de Holanda, Phys. Rev. D 89, 053002 (2014).

[43] R. L. N. Oliveira, Eur. Phys. J. C 76, 417 (2016).

[44] G. Balieiro Gomes, M. M. Guzzo, P. C. de Holanda, and R. L. N. Oliveira, Phys. Rev. D 95, 113005 (2017).

[45] J. A. B. Coelho and W. Anthony Mann, Phys. Rev. D 96, 093009 (2017).

[46] J. A. B. Coelho, W. Anthony Mann, and S. S. Bashar, Phys. Rev. Lett. 118, 221801 (2017).

[47] A. Capolupo, S. M. Giampaolo, and G. Lambiase, Phys. Lett. B 792, 298 (2019).

[48] P. Coloma, J. Lopez-Pavon, I. Martinez-Soler, and H. Nunokawa, Eur. Phys. J. C 78, 614 (2018).

[49] P. Kurashvili, K. A. Kouzakov, L. Chotorlishvili, and A. I. Studenikin, Phys. Rev. D 96, 103017 (2017).

[50] A. Popov and A. Studenikin, Eur. Phys. J. C 79, 144 (2019).

[51] R. Fabbricatore, A. Grigoriev, and A. Studenikin, J. Phys. Conf. Ser. 718, 062058 (2016).

[52] P. Pustoshny and A. Studenikin, Phys. Rev. D 98, 113009 (2018).

[53] D. A. Garanin, Phys. Rev. B 55, 3050 (1997).

[54] K. Fujikawa and R. Shrock, Phys. Rev. Lett. 45, 963 (1980).

[55] A. Loureiro et al., Phys. Rev. Lett. 123, 081301 (2019).

[56] M. Aker et al. (KATRIN Collaboration), Phys. Rev. Lett. 123, 221802 (2019).

[57] J.-S. Lu, Y.-F. Li, and S. Zhou, Phys. Rev. D 94, 023006 (2016).

[58] H.-L. Li, Y.-F. Li, M. Wang, L.-J. Wen, and S. Zhou, Phys. Rev. D 97, 063014 (2018).

[59] K. Abe et al. (Hyper-Kamiokande Proto-Collaboration), arXiv:1805.04163.

[60] A. G. Rosso, F. Vissani, and M. C. Volpe, J. Cosmol. Astropart. Phys. 04 (2018) 040.

[61] S. Seadrow, A. Burrows, D. Vartanyan, D. Radice, and M. Aaron Skinner, Mon. Not. R. Astron. Soc. 480, 4710 (2018). 